Instituto Internacional de Investigación y Desarrollo Tecnológico Educativo INDTEC, C.A. DOI: https://doi.org/10.29394/Scientific.issn.2542-2987.2020.5.15.1.24-43

OAI-PMH: http://www.indteca.com/ojs/index.php/Revista Scientific/oai

Artículo Original / Original Article

\title{
Formación Gerencial Dirigida a Docentes para el Mejoramiento del Proceso Educativo en las Aulas Multigrados
}

\author{
Autora: Adriana Hernández Lobo \\ Universidad Pedagógica Experimental Libertador, UPEL \\ fatimavalentina 22@hotmail.com \\ Mérida, Venezuela \\ https://orcid.org/0000-0003-4974-3465
}

Resumen

La investigación tuvo como propósito diseñar una propuesta de Formación Gerencial para el mejoramiento del Proceso Educativo en las Aulas Multigrados del Núcleo Escolar Rural 442 del municipio Aricagua Estado Mérida. Por lo tanto, se enmarcó en un enfoque cuantitativo, de campo descriptivo, bajo la modalidad de proyecto factible, en una población conformada por quince docentes y un directivo. Para la recolección de la información se elaboró un cuestionario conformado por veintidós preguntas de estructura cerrada, bajo la técnica de la observación. La validez del instrumento se obtuvo a través del juicio de expertos, obteniendo un Coeficiente de Proporción de Rango de 0.96 indicando excelente validez y para la confiabilidad se aplicó la fórmula del Coeficiente de Alfa de Cronbach, cuyo resultado fue 0.96 para ambos estratos de la población. Valorados los resultados se pudo concluir, la necesidad que presentan los docentes de aulas multigrados de obtener una capacitación gerencial que les permita aplicar las funciones gerenciales en el proceso de enseñanza y aprendizaje, estrategias y roles de una población heterogénea donde se atienden diferentes grados e intereses. Por lo que se diseñó, aplicó y evaluó una propuesta dirigidas a docentes y directivo, lográndose fortalecer los conocimientos sobre acciones gerenciales.

Palabras clave: formación; docente; aula.

Cómo citar este artículo:

Hernández, A. (2020). Formación Gerencial Dirigida a Docentes para el Mejoramiento del Proceso Educativo en las Aulas Multigrados. Revista Scientific, 5(15), 24-43, e-ISSN: 2542-2987. Recuperado de: https://doi.org/10.29394/Scientific.issn.2542-2987.2020.5.15.1.24-43

Fecha de Recepción: 26-07-2019
Fecha de Aceptación:

21-11-2019
Fecha de Publicación: 05-02-2020 


\title{
Management Training Directed to Teachers for the Improvement of the Educational Process in Multigrade Classrooms
}

\begin{abstract}
The purpose of the research was to design a proposal for Management Training for the improvement of the Educational Process in the Multigrade Classrooms of the Rural School Center 442 of the Aricagua State Mérida municipality. Therefore, it was framed in a quantitative, descriptive field approach, under the feasible project modality, in a population made up of fifteen teachers and one manager. For the collection of the information, a questionnaire was prepared consisting of twenty-two closed-structure questions, under the observation technique. The validity of the instrument was obtained through expert judgment, obtaining a Range Proportion Coefficient of 0.96 indicating excellent validity and for reliability the Cronbach's Alpha Coefficient formula was applied, whose result was 0.96 for both strata of the population. Once the results have been assessed, the need for teachers of multigrade classrooms to obtain a managerial training that allows them to apply the management functions in the teaching and learning process, strategies and roles of a heterogeneous population where different degrees and interests are attended. For what was designed, applied and evaluated a proposal aimed at teachers and managers, managing to strengthen knowledge about management actions.
\end{abstract}

Keywords: training; teacher; classrooms.

\section{How to cite this article:}

Hernández, A. (2020). Management Training Directed to Teachers for the Improvement of the Educational Process in Multigrade Classrooms. Revista Scientific, 5(15), 24-43, e-ISSN: 2542-2987. Recovered from: https://doi.org/10.29394/Scientific.issn.2542-2987.2020.5.15.1.24-43

Date Received:

26-07-2019
Date Acceptance:

21-11-2019
Date Publication: 05-02-2020 


\section{Introducción}

La formación profesional está relacionada con los conocimientos, habilidades y preparación que posee una persona para ejercer un oficio o profesión. Son los saberes y competencias que potencian el desarrollo socio personal e institucional. Por tanto, la formación constituye los estudios y aprendizajes permanentes encaminados a la actualización profesional en un proceso de construcción que se va desarrollando en función de las exigencias sociales planteadas por el mundo globalizado y tecnificado actual.

En este contexto, la formación docente representa ese proceso educativo mediante el cual, se perfecciona la teoría y la praxis de áreas específicas del conocimiento en sintonía con las exigencias personales y requerimientos profesionales del ejercicio de la docencia, para que el docente se convierta en un transformador de su propia realidad solucionando los problemas educativos en las manifestaciones cognitivas, afectivas $y$ psicomotoras que presentan los estudiantes.

Bajo estas premisas, Contreras y Mujica (2014a), exponen que: "para el docente y su proceso de formación el reto resulta de mayor responsabilidad e importancia, ya que debe enfrentarse a diversos escenarios que requiere la reflexión sobre su propia práctica" (pág. 52); por ello, debe desarrollar el pensamiento crítico en función de las situaciones de su entorno social que le ayuden a él y a su comunidad a superar las debilidades encontradas.

Desde esta perspectiva, la formación gerencial del docente constituye un aspecto importante, en relación a los procesos administrativos de planificar las acciones y estrategias, coordinar los recursos, organizarlos, ejercer dirección y control con el fin de lograr los objetivos y metas preestablecidos. En tal sentido, un gerente tiene que conjugar una serie de factores para impulsar el éxito personal, profesional y organizacional. La actuación del docente como gerente educacional está relacionada con los saberes en planificación, organización y evaluación. 
Al respecto, Calvo (2013): expresa, que en el término de inclusión educativa se "ha entrado con fuerza en el discurso político-pedagógico, en un contexto en que simultáneamente se profundizan los procesos de exclusión y se reconocen las dificultades institucionales y pedagógicas para lograr las metas de una educación de calidad para todos" (pág. 23); pero la realidad es otra y se ha demostrado que tender un puente entre el discurso y los hechos no es algo sencillo.

A tal efecto, la gerencia educativa se asume como la fuerza que impulsa la eficacia en el trabajo con la participación de los entes involucrados (padres, madres y comunidad) para mejorar el desarrollo socio cultural del estudiante y el entorno donde se encuentra la institución. El docente como gerente debe tener la capacidad y responsabilidad de dirigir el proceso de enseñanza y aprendizaje con optimismo estimulando el aprender- aprender y querer seguir aprendiendo con experiencias significativas.

Con referencia a lo anterior, la labor educativa en las aulas multigrados ubicadas en los sectores rurales demanda de docentes con formación gerencial que planifiquen, organicen, controlen y evalúen el proceso de enseñanza y aprendizaje en una población escolar de diferentes grados con diversos currículos, intereses, necesidades y ritmos de aprendizaje que amerita poseer competencias gerenciales de planificador, líder, motivador, tomador de decisiones, comunicador y agente de cambio, entre otros, para hacer que el proceso de enseñanza aprendizaje sea productivo.

De acuerdo con lo anteriormente expuesto, Contreras y Mujica (2014b), establecen que:

La escuela en el sector rural representa el mejor escenario para experimentar el desarrollo de competencias que satisfagan los requerimientos de las comunidades y la aplicación de políticas educativas públicas, con un currículo permeable, flexible, constructivo y con pertinencia social (pág. 44). 
Por tal motivo, el docente debe convertir la escuela en una opción atractiva para que los niños y niñas reciban una educación pertinente a la realidad social. Para cumplir con esta misión el docente de aula debe ser un gerente que promueva la participación y trabajo en equipo, liderice un proceso educativo de integración en el cual se relacionen los conocimientos de los estudiantes con los contenidos a aprender. En esta concepción el gerente de aula analiza e interpreta las situaciones de aprendizajes y toma decisiones de acuerdo a las características del contexto y educando.

Desde esta perspectiva el docente de aulas multigrado debe ser conductor de condiciones favorables para la construcción de aprendizajes, que favorezca la participación y el deseo de seguir aprendiendo. Asimismo, promueva la reflexión para establecer los criterios de cambios de la acción educativa en función de las necesidades e intereses de los estudiantes, en un proceso coherente con visión de conjunto sistemático que propicie la adquisición del conocimiento.

En este contexto, en el municipio Aricagua ubicado al sur del estado Mérida se encuentra el Núcleo Escolar Rural 442, conformado por ocho (8) planteles que concentran varias aulas multigrados, específicamente de primero a tercer y de cuarto a sexto grado de Educación primaria a cargo de dos docentes que cumplen funciones pedagógicas y administrativas. Cabe resaltar que los docentes carecen de formación gerencial para lograr las metas y objetivos educativos, presentan debilidades para planificar, organizar, controlar y evaluar las áreas curriculares, tampoco reciben acompañamiento pedagógico que oriente su acción educativa.

Igualmente, los docentes necesitan conocimientos gerenciales relacionados con las estrategias de comunicación, motivación, liderazgo, trabajo en equipo y la toma de decisiones en consenso que motiven a los padres y representantes a colaborar con la formación integral del estudiante. Asimismo, carecen de recursos didácticos para desarrollar algunos contenidos 
curriculares y falta estimulo en el estudiante para obtener mejor rendimiento escolar.

De igual manera, el docente de aula multigrado necesita formación académica para integrar los contenidos curriculares en función de los diferentes grados considerando los intereses y necesidades de los estudiantes. Por otra parte, los padres y representantes poco colaboran con las actividades educativas. Esto trae como consecuencia una praxis educativa descontextualizada, no significativa y poco estimulante al trabajo cooperativo en las aulas multigrado, que no considera los intereses y necesidades de cada estudiante para orientarlo en su proceso formativo.

Ante esta situación surgen como interrogantes dentro de la investigación: ¿Cuál es la formación gerencial que poseen los docentes de las aulas multigrados?; ¿Qué importancia tiene la formación gerencial en el quehacer educativo?; ¿Es factible aplicar una propuesta de formación gerencial a los docentes de las aulas multigrados para el mejoramiento del proceso educativo?; ¿Qué beneficios proporcionará a los docentes de las aulas multigrados la propuesta gerencial?

En concordancia con las interrogantes surgidas la investigación formula como objetivo para orientar la problemática objeto de estudio: proponer un plan de acción para la formación gerencial dirigida a los docentes para el mejoramiento del proceso educativo en las aulas multigrados de las escuelas rurales del Núcleo Escolar Rural 442 municipio Aricagua del estado Mérida.

\subsection{La Gerencia en el campo educativo}

En términos generales la gerencia es la acción de conducir, dirigir, orientar, persuadir, interactuar y delegar acciones que van a permitir a los seres humanos asumir el control de sus vidas, responsabilizándose de su proceder para ejercer el manejo de las organizaciones y el buen funcionamiento de las mismas. Al respecto, Carrillo (2016), señala que la 
gerencia es: "la ventana que abre nuevas perspectivas a las organizaciones actuales, amerita de gerentes fortalecedores de los canales participativos para la intervención activa de los trabajadores, al momento que identifican prioridades y formulan objetivos, además de ejercer un adecuado control sobre la organización" (pág. 139). Se infiere, que todo proceso gerencial implica el manejo de recursos humanos y materiales que se combinan de forma organizada en función de obtener resultados determinados.

En atención a lo antes expuesto, en el campo educativo, Barrios (2018), establece que: "toda organización con aspiraciones de excelencia debería estar enfocada en ampliar el conocimiento, en emplear métodos, normas, valores, lealtad; que permita enfrentar la realidad, adaptarse al contexto cambiante, complejo e incierto" (pág. 217). Al respecto, se entiende que la gerencia es esencial en la administración de las instituciones y está vinculada con las actividades escolares, la cual debe adaptarse a las situaciones actuales, a los cambios socioeducativos, culturales, económicos, políticos, tecnológicos del país. Por lo tanto, los procesos educativos necesitan ser conducidos por verdaderos gerentes, preparados en el área y seguros del rol que desempeñan en la educación venezolana.

Para lograr una eficiente gerencia educativa, es necesario que haya buenos gerentes educativos, es decir, que el recurso humano esté capacitado y dispuesto a ejecutar las funciones gerenciales como tal: planificar, organizar, dirigir, controlar, interactuar, interrelacionar el proceso administrativo, ponerlo en acción, generar cambios, dinamizar el proceso, darle utilidad y calidad al quehacer educativo.

Desde este escenario, Granado (2016), plantea que: "se vislumbra el requerimiento de un gerente educativo con las competencias idóneas para el cumplimiento cabal de sus funciones, que involucre al personal que esté bajo su cargo, a través del seguimiento en los diferentes procesos" (pág. 183). De acuerdo a esta consideración, la función principal de la gerencia educativa es 
lograr la integración social del colectivo y formar un recurso humano integro, en un ambiente agradable, acorde a sus necesidades, dentro de un proceso organizado donde se pueda interactuar y compartir en los aspectos técnicos, pedagógicos, económicos, académicos que aseguren el logro de un desempeño eficaz y eficiente en la institución.

\subsection{Funciones del Gerente Educativo}

La gerencia es uno de los aspectos importante en el funcionamiento de las instituciones educativas, por ende, el director como gerente educativo de la institución y los docentes como gerentes de aula deben prepararse y actualizarse para adquirir conocimientos que le permitan ejecutar eficazmente sus funciones. En tal sentido, el gerente educativo en el desarrollo de su gestión, debe seguir un proceso universal que contribuya al desarrollo con el propósito de continuar en la búsqueda permanente de excelencia y calidad.

En este orden de ideas, la gerencia permite la generación y difusión de conocimientos orientados en la preparación de los alumnos, para que enfrenten la vida como ciudadanos de alta participación en el desarrollo económico y social de su comunidad, trátese de una comunidad rural o no, y su incorporación futura al campo de trabajo con el conjunto de competencias requeridas por un mundo cada vez más interdependiente y de alta competencia.

En concordancia con lo anterior, Salazar (2003), manifiesta: "todo gerente educativo debe emprender un esfuerzo que asegure un buen resultado en el funcionamiento de su institución a través del trabajo cooperativo y la toma de decisiones consensuadas sobres las acciones a ejecutar en la organización escolar" (pág. 126). De lo expuesto, cabe destacar el gerente educativo mejora la calidad de los procesos de trabajo (técnicas de enseñanza - aprendizaje y el trabajo administrativo), fomentando la excelencia en la enseñanza, propiciando el trabajo en equipo y escuchando los 
planteamientos de los demás entes involucrados.

De acuerdo con lo anterior, todo proceso gerencial implica una serie de funciones que para Sallenave (2006), consta de: Planeación, Organización, Dirección y Control (pág. 235).

Planeación: consiste en la toma de decisión entre gerente y equipo para decidir el camino más acertado a fin de transformar la institución y alcanzar la excelencia pedagógica en función del proyecto educativo institucional y de aula, a partir del diagnóstico de la realidad, fijación de objetivos, acciones a seguir y recursos asignados.

Organización: Implica el diseño de la estructura formal para el desarrollo de la gestión, facilita la integración y coordinación de actividades de todos los agentes involucrados en el proceso educativo; empleo de recursos, proyectos, programas, procesos que involucre la segmentación de las funciones y labores por medio de una escala jerárquica.

Dirección: consiste en la instauración de un clima organizacional por parte del directivo, que comprenda elementos clave de liderazgo, motivación e integración de las habilidades y potencialidades de los actores; lo cual permite establecer una relación directa y un compromiso auténtico con el proyecto educativo a fin de optimizar la práctica educativa.

Control y evaluación: Es necesario para asegurar la ejecución de la programación de acuerdo al esquema de responsabilidades y distribución del trabajo para lograr los objetivos y metas propuestas. Para lo cual se deben establecer criterios y sistemas de información para evaluar, retroalimentar el avance y logro de los objetivos a fin de tomar decisiones y reprogramar de ser necesario.

\subsection{El Docente como Gerente de Aula}

El docente como gerente de aula ejecuta o implementa una serie de roles gerenciales como son: planificador, líder, motivador, tomador de 
decisiones, comunicador, agente de cambios, entre otros, para lo cual requiere de una formación gerencial amplia y suficiente para poder desempeñarse en el aula y hacer que su proceso de enseñanza aprendizaje sea productivo.

De acuerdo con el Ministerio del Poder Popular para Educación, Cultura y Deportes (2007), el docente como gerente de aula debe reunir las siguientes características:

Promotor y líder de la institución a fin de enriquecer la práctica pedagógica. Abierto al cambio, emocionalmente seguro de lo que persigue. Impartir una educación significativa orientada hacia los problemas sociales, éticos y morales del entorno. Formar individuos con mentalidad amplia, sensible, analítica, crítica, capaces de hacer, crear y dispuestos a servir a la humanidad, respetando sus valores autóctonos. Desarrollar una educación bajo un punto de vista constructivista para ayudar al educando de manera progresiva y secuencial, tomar en cuenta su edad cronológica y su desarrollo para la construcción de su conocimiento, propiciar experiencias de aprendizaje en el aula y fuera de ella para así estimular actividades indagadoras (pág. 24).

Estas características, definen el perfil académico y profesional que se espera lograr en el docente, a través de prácticas gerenciales relacionadas con el interaprendizaje de la metodología de la investigación, actualmente enmarcada en el Sistema Educativo Bolivariano, que requiere del rol de un docente líder en la escuela y en la comunidad, centrado en orientar la solución de sus problemas, al mismo tiempo promover en sus miembros actitudes analíticas y reflexivas en torno al medio ambiente donde se desenvuelven.

\subsection{Aulas multigrados}

El aula multigrado es una consecuencia de las escuelas unidocentes y de las escuelas multigrado. En otras palabras, cuando un educador atiende un aula multigrado se refiere a atender varios grados al mismo tiempo en el mismo 
espacio educativo (aula), y, mientras más grados atienda el docente, más complicado se hace el trabajo, difícil de controlar, mayor responsabilidad entre otros; es posible en una Institución Educativa primaria siendo escuela multigrado, algunos de los docentes laboren como si se encontraran en aulas mono grado (1 solo grado).

En tal sentido, Padawer (2008), expresa que: "las aulas multigrado o polígrado responden a una matrícula pequeña de estudiantes con diferentes edades y niveles académicos, atendidas por un maestro en una sala, generalmente se ubican en regiones territoriales aisladas" (pág. 157). En otras palabras, aulas que funcionan con un grupo pequeño de estudiantes los cuales poseen un nivel académico diferente y por encontrarse en condiciones geografías muy aisladas son atendidas por un docente.

\section{Metodología}

La investigación respondió a la modalidad de proyecto factible, apoyado en el estudio de campo de carácter descriptivo, bajo el paradigma cuantitativo. En tal sentido, el proyecto factible de acuerdo con la Universidad Pedagógica Experimental Libertador (UPEL, 2016), consiste en: "la investigación, elaboración y desarrollo de una propuesta de un modelo operativo viable para solucionar un problema, requerimientos o necesidades de organización o grupos sociales; puede referirse a la formulación de las políticas, programas, tecnologías, métodos o procesos" (pág. 21).

En este caso el producto final, consiste en la propuesta de formación gerencial dirigida a docentes de aulas multigrados, la cual puede ser implementada en cualquier escuela que presente aulas de este tipo. La investigación se realizó en las instituciones educativas en el Núcleo Escolar Rural 442 del municipio Aricagua del estado Mérida, con la finalidad de proporcionar formación gerencial a los docentes para contribuir en el desempeño eficiente de su praxis diaria. 
Asimismo, se apoyó en una investigación de campo, la cual es definida por Arias (2012a): como "aquella que consiste en la recolección de datos directamente de los sujetos investigados, o de la realidad donde ocurren los hechos (datos primarios), sin manipular o controlar alguna variable" (pág. 31); lo que permitió obtener información directa y efectiva de los docentes que laboran en las aulas multigrados pertenecientes a las instituciones educativas del Núcleo Escolar Rural 442 municipio Aricagua del estado Mérida.

También, fue de carácter descriptivo porque busca caracterizar el tema en estudio, con el propósito de estructurarlo. Al respeto, Hurtado (2010), refiere que la investigación descriptiva: "tiene como objetivo la descripción precisa del evento de estudio, haciendo una enumeración detallada de sus características" (pág. 101); o sea, las dimensiones que forman parte de las variables comprendidas en el estudio, para ello se escogió la información pertinente y se valoró de manera independiente, para luego poder describir lo investigado.

De igual manera la investigación se apoyó en el paradigma cuantitativo, tal como lo explica Hernández, Fernández y Baptista (2010): "la investigación cuantitativa usa la recolección de datos para probar hipótesis, con base en la medición numérica y el análisis estadístico, para establecer patrones de comportamiento y probar teorías" (pág. 4); dicho de otra manera, se recogen y analizan los datos sobre las variables para llegar a establecer un análisis cuantitativo. Asimismo, Sabino (2003), refiere:

Este tipo de operación se efectúa, naturalmente, con toda la información numérica resultante de la investigación. Ésta luego del procedimiento de análisis, se presentará en un conjunto de cuadros, tablas y medidas, a las cuales se les ha calculado los porcentajes y presentado convenientemente (pág. 134).

Los datos recopilados del cuestionario aplicado a docentes y directivos se sometieron a análisis estadísticos a fin de calcular frecuencias, estadísticos 
descriptivos y la elaboración de gráficos de barra, en los cuales se presentó el promedio de la media aritmética obtenida de las respuestas aportadas por la muestra objeto de estudio a los indicadores que conforman las preguntas que dan respuestas a la problemática encontrada.

\subsection{Técnicas e instrumentos de recolección de datos}

Para la recolección de datos se utilizó la encuesta, a través de un instrumento tipo cuestionario. La técnica según Arias (2012b): "es el procedimiento o forma particular de obtener datos o información" (pág. 67); ésta utiliza como instrumento el cuestionario, el cual es definido por el mismo autor Arias (2012c), como: "la herramienta en cuya estructura quedan registradas las respuestas suministradas por el encuestado (pág. 69).

Por lo tanto, se diseñó un cuestionario cerrado con veintidós (22) ítems tipo escala Likert de cinco opciones de respuesta: Siempre, Muchas Veces, Pocas Veces, Rara Vez y Nunca. La distribución de los ítems se corresponde con los indicadores: crecimiento profesional, aplica las funciones gerenciales, utiliza estrategias gerenciales, interrelación de contenidos curriculares, trabajo grupal, promoción de la participación y cooperación y desarrollo del potencial humano. Correspondientes a las diferentes dimensiones y variables que conforman el estudio.

\subsection{Resultados de la investigación}

En base a los resultados obtenidos, al aplicar el cuestionario al directivo y docentes de las instituciones educativas del Núcleo Escolar Rural 442, municipio Aricagua del estado Mérida, se llegó a las siguientes conclusiones, que se aprecian en la tabla 1 y se explican a continuación: 
Tabla 1. Resultados obtenidos del cuestionario aplicado por la investigadora.

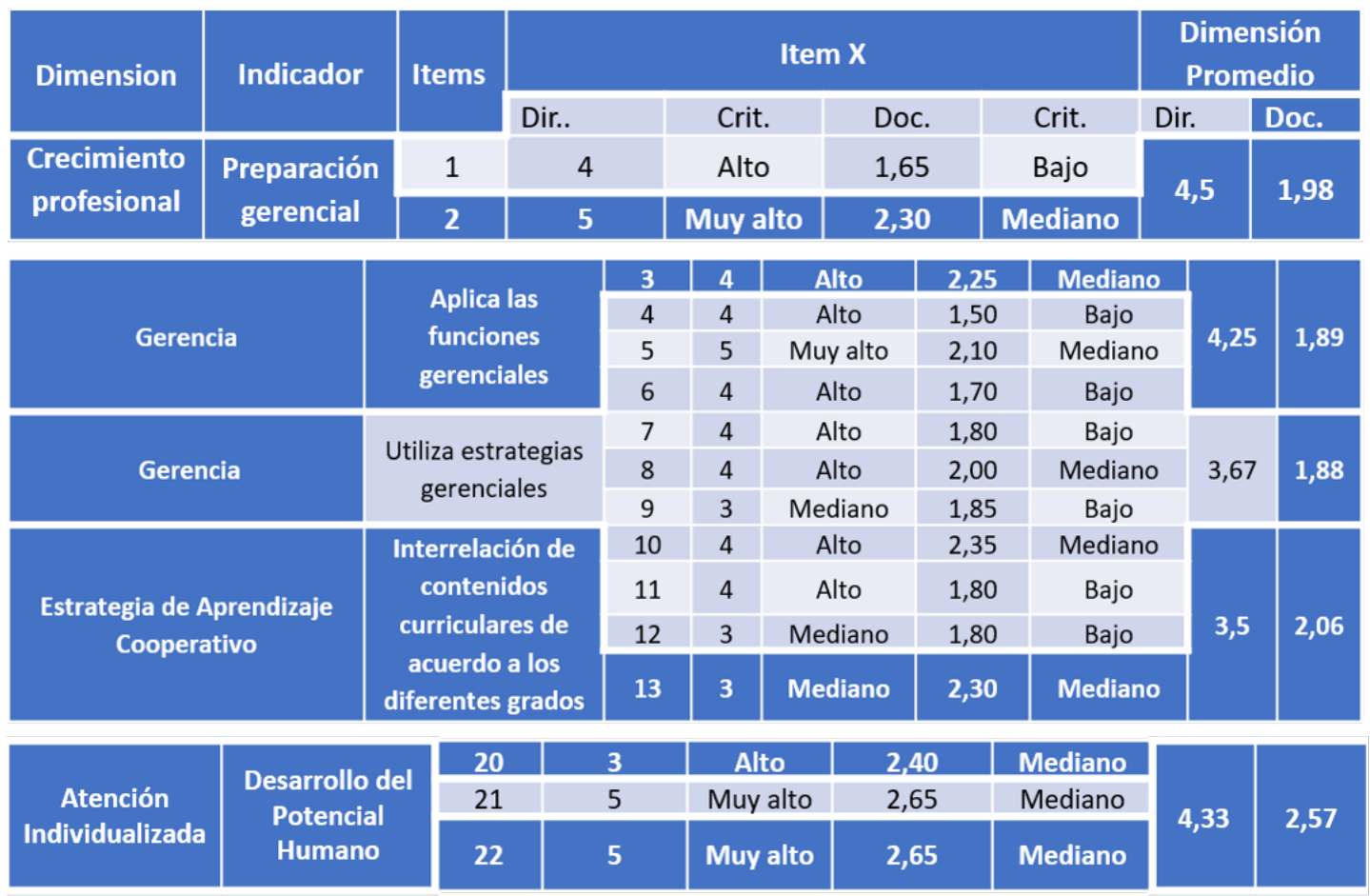

Fuente: La Autora (2019).

En el indicador, preparación gerencial, dimensión crecimiento profesional, los docentes carecen de formación gerencial para tener un desempeño eficiente y eficaz. En cuanto, al indicador, aplica funciones gerenciales y dimensión gerencia, los docentes expresaron no aplicar las funciones gerenciales de planificación, organización, control y evaluación en el proceso de enseñanza y aprendizaje del aula multigrado.

En relación, al indicador, estrategias gerenciales, dimensión gerencia, los docentes expresaron que existe poca comunicación con los entes escolares y algunas veces participan en la toma de decisiones en la institución. Con respecto, al indicador, interrelación de los contenidos curriculares, dimensión gerencia, los docentes manifiestan no recibir ningún tipo de orientación para desarrollar los contenidos curriculares en las aulas 
multigrados que comprende diversos grados, intereses, necesidades y expectativas en relación a la población escolar.

Así mismo, el indicador, trabajo grupal, dimensión estrategias de aprendizaje cooperativo, los docentes expresaron utilizarla algunas veces. De igual manera, el indicador promoción de la participación y cooperación, dimensión estrategia de aprendizaje cooperativo, los docentes expresaron que algunas veces utilizan el trabajo cooperativo en las aulas multigrado. Por otra parte, el indicador desarrollo del potencial humano, dimensión atención individualizada, manifestaron algunas veces utilizan la atención individualizada para resolver las necesidades de la población escolar.

A fin de dar respuestas a las necesidades detectadas y señaladas anteriormente producto del trabajo de campo realizado en el Núcleo Escolar Rural 442, municipio Aricagua del estado Mérida, constituyen la base para diseñar el plan de acción para resolver o mejorar la situación detectada. Por lo tanto, la propuesta está enmarcada hacia la formación gerencial de los docentes para que optimicen su desempeño laboral, con la finalidad de mejorar el proceso de enseñanza y aprendizaje en las aulas multigrados.

Del mismo modo, se pretende que el docente se apropie de estrategias para desarrollar el trabajo de las aulas multigrados, entre las que se mencionan la atención individualizada y el trabajo cooperativo; realizando control y seguimiento a los logros obtenidos con la aplicación de las funciones y estrategias gerenciales.

\section{Conclusiones}

En el diagnóstico realizado se pudo evidenciar que los docentes no reciben formación gerencial de parte del directivo, induciéndolos al desconocimiento de los elementos relacionados con la funciones y estrategias gerenciales, aspectos que deben estar presentes en las acciones aplicadas por el docente como gerente de aula para proporcionar aprendizajes 
significativos en los estudiantes y a su vez optimizar el proceso de enseñanza y aprendizaje en las aulas multigrados. De igual manera, se determinó que los docentes carecen de conocimientos para interrelacionar los contenidos curriculares de acuerdo a los diferentes grados, necesidades e intereses de los estudiantes y no reciben orientaciones del personal directivo sobre estrategias innovadoras para organizar los contenidos curriculares en el aula multigrados.

Por otra parte, la mayoría de los docentes no aplican la técnica de trabajo grupal como estrategia importante para incrementar el rendimiento académico de los escolares y fortalecer la responsabilidad grupal. Del mismo modo, no promueven el trabajo cooperativo para fomentar la participación, desarrollar la colaboración y fortalecer la valoración entre compañeros. Asimismo, es necesario que los docentes empleen la estrategia de la atención individualizada en el aula de clase para resolver las necesidades y debilidades de cada estudiante, permitiendo desarrollar el potencial humano.

En este orden de ideas, el aprendizaje cooperativo se concibe como un enfoque pedagógico que consiste en organizar las actividades escolares a modo de convertirlas en aprendizajes y experiencias sociales, para ello es necesario que los estudiantes realicen las actividades dentro de aula de clase de forma grupal o colectiva a través del intercambio de información.

Del mismo modo, en el aula multigrado se tiene la particularidad de estudiantes con diversidad de grados, edades y ritmos de trabajo, por ello, se hace necesario atender las necesidades de manera individualizada, aplicando la estrategia de la educación personalizada, la cual permite ayudar a cada estudiante a superar las debilidades y satisfacer las necesidades escolares, lo cual implica toda la dedicación, ayuda, mediación y orientación necesaria del docente para cada alumno logrando desarrollar al máximo todo su potencial humano.

En este sentido, las acciones gerenciales desarrolladas permitieron 
ayudar a elevar de alguna manera la acción pedagógica del docente, por cuanto asuman con seriedad la necesidad de capacitarse y actualizarse en relación a las diferentes técnicas, métodos y estrategias gerenciales que contribuyen a la formación integral del estudiante, por ello, se aplicó dentro de la investigación un conjunto de actividades enmarcadas en la formación gerencial del docente del aula multigrado.

Así mismo, el docente del aula multigrado necesita una formación especial, por cuanto debe desenvolverse en un ambiente de trabajo con características particulares, atendiendo diversidad de grados, intereses y necesidades, por eso el maestro rural debe ejercer una labor abnegada para convertirse en un modelo para sus estudiantes y la comunidad en general. Visto de esta manera, ese es el camino que debe seguir todo docente, hacia una actitud investigativa, dispuesto al mejoramiento de su profesión, cuya labor y misión responda a los requerimientos de su contexto social, solo se requiere el cambio de actitud.

La propuesta de las acciones planificadas son una alternativa que debe ser seleccionada con una visión prospectiva, por cuanto proporcionará los mayores beneficios a los docentes, al capacitarlos en la aplicación de acciones, estrategias y roles gerenciales que contribuyen a mejorar el desempeño laboral buscando que la Institución se perpetúe y se desarrolle con el propósito de continuar en la búsqueda permanente de excelencia y calidad, en la generación y difusión de conocimientos.

Por consiguiente, la formación de los docentes conduce al mejoramiento del proceso enseñanza y aprendizaje haciéndolo eficiente y de impacto significativo en el bienestar social de las comunidades; igualmente, deben considerarse la participación ciudadana como primordial en la organización, la toma de decisiones y solución de problemas contribuyendo de esta manera con el desarrollo de la comunidad. 


\section{Referencias}

Arias, F. (2012a,b,c). El proyecto de investigación. Introducción a la metodología científica. Caracas, Venezuela: Editorial Espíteme, págs. 9-143.

Barrios, R. (2018). Cultura y Valores Organizacionales Emergentes en la Gerencia Transcompleja. Revista Scientific, 3(9), 214-232, e-ISSN: 2542-2987. Recuperado de:

https://doi.org/10.29394/Scientific.issn.2542-2987.2018.3.9.11.214-232

Calvo, G. (2013). La formación de docentes para la inclusión educativa. Páginas de Educación, 6(1), 19-35, e-ISSN: 1688-7468. Recuperado de: http://www.scielo.edu.uy/scielo.php?script=sci arttext\&pid=S1688$\underline{74682013000100002}$

Carrillo, M. (2016). Gerencia y Sociedad del Conocimiento para Optimizar el Desempeño Académico del Docente. Revista Scientific, 1(1), 134149, e-ISSN: 2542-2987. Recuperado de:

https://doi.org/10.29394/scientific.issn.2542-2987.2016.1.1.8.134-149

Contreras, W., \& Mojica, G. (2014a,b). El docente del sector rural y sus competencias. 1era edición. Caracas, Venezuela: Serie de libros arbitrados del Vicerrectorado de Extensión de la Universidad Pedagógica Experimental Libertador. Recuperado de:

http://espaciodigital.upel.edu.ve/bitstream/123456789/460/2/\%20\%20E \%20Docente\%20del\%20Sector\%20Rural\%20y\%20sus\%20Competen cias\%20.pdf

Granado, N. (2016). Arte de ser Gerente Educativo en la actualidad. Revista Multidisciplinaria Dialógica, 13(1), 180-201. ISSN: 1690-8961; e-ISSN: 2244-7662. Recuperado de:

http://revistas.upel.edu.ve/index.php/dialogica/article/view/4824

Hernández, R., Fernández, C., \& Baptista, P. (2010). Metodología de la investigación. 5ta edición. México: McGraw-Hill, págs. 2-599. 
Hurtado, J. (2010). El proyecto de investigación. 6ta edición. Caracas, Venezuela: Editorial Quirón, págs. 7-181.

Ministerio del Poder Popular para Educación, Cultura y Deportes (2007). Manual del Docente. Caracas, Venezuela: Centro Nacional para el Mejoramiento de la Enseñanza de la Ciencia - CENAMEC.

Padawer, A. (2008). Cuando los grados hablan de desigualdad. Primera edición. Buenos Aires, Argentina: Editorial Teseo.

Sabino, C. (2003). El proceso de la investigación. Nueva edición actualizada. Caracas, Venezuela: Editorial Panapo.

Salazar, R. (2003). El nuevo gerente venezolano. Venezuela: Ediciones OPSU.

Sallenave, J. (2006). Gerencia y planeación estratégica. Colombia: Editorial Norma, S.A.

UPEL (2016). Manual de Trabajos de Grado de Especialización y Maestría y Tesis Doctorales. 5ta Edición, ISBN: 980-273-441-1. Caracas, Venezuela: Fondo Editorial de la Universidad Pedagógica Experimental Libertador, FEDUPEL. 


\section{Adriana Hernández Lobo \\ e-mail: fatimavalentina 22@hotmail.com}

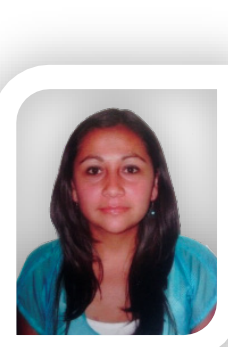

Nacida en Mérida estado Mérida, Venezuela, el 22 de junio del año 1988. Licenciada en Educación, egresada de la Universidad Bolivariana de Venezuela (UBV); Especialista en Gerencia Educacional en la Universidad Pedagógica Experimental Libertador (UPEL); Docente de aula multigrado en el sector rural, municipio Aricagua del estado Mérida; Facilitadora de seminario I, en la maestría de Gerencia Educativa de la Universidad Pedagógica Experimental Libertador (UPEL). 